\title{
Peut-on toucher aux droits des pa- tients par des limitations tarifaires?
}

\section{Dr Urs Stoffel}

membre du Comité central, responsable du département Médecine et tarifs ambulatoires

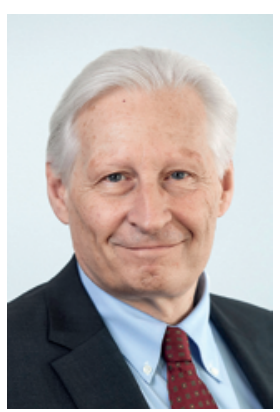

La réponse est simple: les partenaires tarifaires ne peuvent pas limiter, dans un tarif, le droit des patients aux prestations inscrit dans la loi. Encore moins, si le seul et unique but visé est d'empêcher une augmentation du volume de prestations. Pour cela, d'autres bases et mécanismes légaux existent depuis l'introduction du TARMED.

La loi sur l'assurance-maladie (LAMal), qui constitue le fondement du tarif ambulatoire TARMED, se base sur la présomption des prestations obligatoires, c'est-à-dire que le traitement médical répond par principe aux critères d'efficacité, d'adéquation et d'économicité (critères EAE), tant que le Conseil fédéral ne l'exclut pas de l'obligation de prise en charge ou ne le lie pas à certaines conditions. Le patient est donc fondamentalement en droit de bénéficier de ces prestations dites obligatoires.

Le droit des patients à une prestation obligatoire ne peut pas être restreint par une limitation dans le tarif.

Deux avis de droit sur la question du budget global et de la tarification dans l'assurance-maladie ${ }^{1}$ et sur celle des réelles prestations supplémentaires dans le domaine ambulatoire ${ }^{2}$ viennent confirmer l'affirmation selon laquelle les prestations rendues obligatoires par la LAMal ne peuvent pas être restreintes par des limitations dans le tarif.

1 Prof. iur. Ueli Kieser, Zurich/Saint-Gall, «Fragen des Globalbudgets und der Tarifgestaltung in der Krankenversicherung", 2017 (en allemand)

2 Prof. iur. Ueli Kieser, Zurich/Saint-Gall, «Fragen zu echten Mehrleistungen im ambulanten Bereich", 2018 (en allemand)
Le droit des patients à une prestation obligatoire ne peut donc pas être restreint par une limitation dans le tarif. Seul le Conseil fédéral pourrait définir une telle limitation et, le cas échéant, cette dernière ne pourrait pas être fixée directement dans le tarif mais devrait être inscrite dans l'ordonnance sur les prestations de l'assurance des soins (OPAS) et surtout répondre aux critères EAE, puisque que toutes les prestations défi- nies dans l'OPAS doivent respecter ces trois critères, conformément à l'art. 32 LAMal.

L'Office fédéral de la justice (OFJ) va même encore plus loin en affirmant à l'Office fédéral de la santé publique (OFSP) qu'il n'est pas prévu que les fournisseurs de prestations et les assureurs décident ensemble de limiter des prestations obligatoires dans la structure tari-

\section{La FMH rejette catégoriquement toute limita-} tion des prestations au temps dans le tarif ambulatoire dont le seul but est d'empêcher une augmentation du volume.

faire. Il interdit ainsi purement et simplement aux partenaires tarifaires d'introduire des limitations dans le tarif. Là aussi, seul le Conseil fédéral pourrait en introduire, non pas directement dans le tarif, mais uniquement via l'OPAS.

La FMH a toujours été claire à ce sujet, en particulier lors des négociations tarifaires: elle rejette catégoriquement toute limitation des prestations au temps dans le tarif ambulatoire qui a pour seul et unique but d'empêcher une augmentation du volume de prestations et, par conséquent, répond à une motivation purement économique.

A ce sujet, l'un des avis de droit précités est clair. En présence de prestations supplémentaires injustifiées générant une augmentation du volume, la loi a prévu des mécanismes reposant exclusivement sur les critères EAE qui permettent d'éviter les abus et même d'exiger la restitution des sommes concernées (cf. art. 56 LAMal).

La FMH soutient donc les limitations uniquement lorsqu'elles sont médicalement indiquées, édictées sur la base de dispositions légales (p. ex. radioprotection) ou visent à protéger les patients. De telles limitations sont appropriées et bénéficient donc de l'appui de la FMH et de ses organisations affiliées. 